\title{
3D Reconstruction of M11A2 from the SELENIUM Experiment
}

B. D. Miller, M. Abir, A. Aitkaliyeva, A. Leenaers, B. J. Hernandez, W. Van Renterghem, S. Van den Berghe, A. Winston, D. D. Keiser, Jr.

January 2017

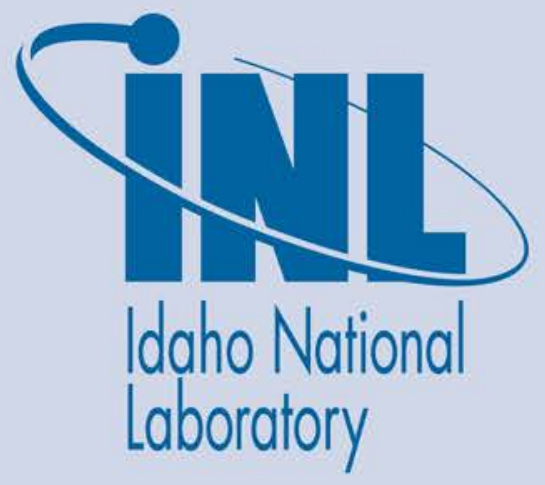

The INL is a U.S. Department of Energy National Laboratory operated by Battelle Energy Alliance 
INL/EXT-17-40936

\title{
3D Reconstruction of M11A2 from the SELENIUM Experiment
}

\author{
B. D. Miller, M. Abir, A. Aitkaliyeva, A. Leenaers, B. J. Hernandez, \\ W. Van Renterghem, S. Van den Berghe, A. Winston, D. D. Keiser Jr.
}

January 2017

\section{Idaho National Laboratory \\ Idaho Falls, Idaho 83415}

http://www.inl.gov

\author{
Prepared for the \\ U.S. Department of Energy \\ Assistant Secretary for _, OR Office of \\ Under DOE Idaho Operations Office \\ Contract DE-AC07-05ID14517
}




\section{D Reconstruction of M11A2 from the SELENIUM Experiment}

B. D. Miller, M. Abir, A. Aitkaliyeva, A. Leenaers, B. J. Hernandez, W. Van Renterghem, S. Van den Berghe, A. Winston, D. D. Keiser Jr. 


\section{Contents}

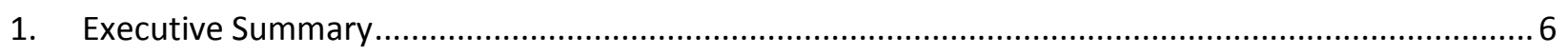

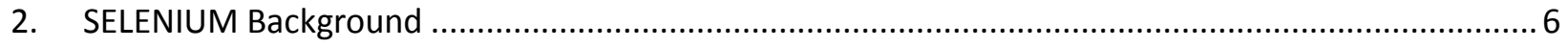

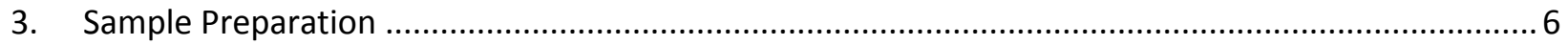

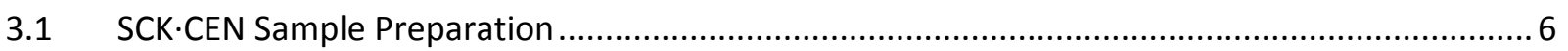

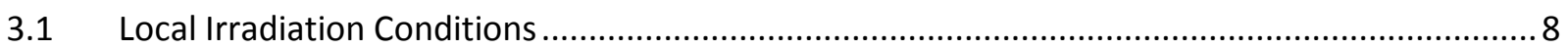

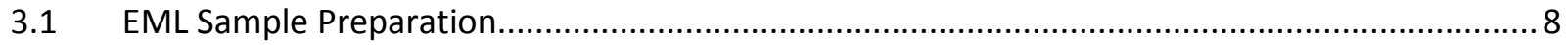

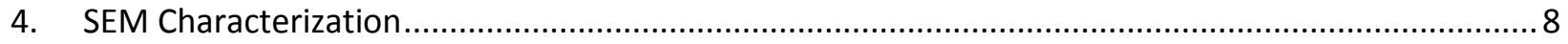

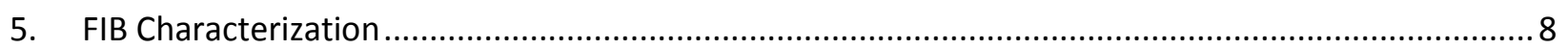

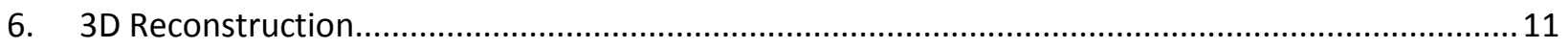

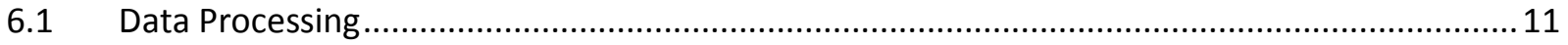

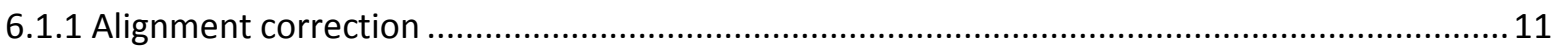

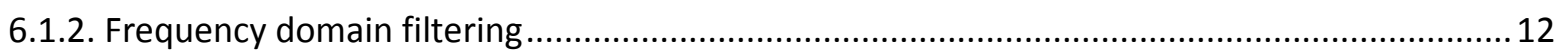

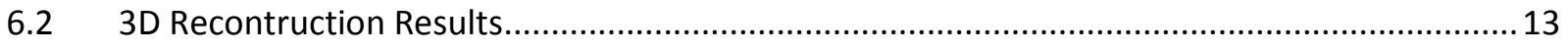

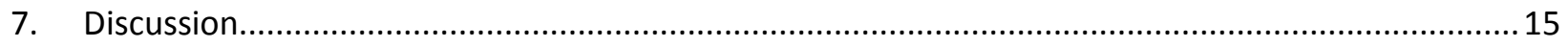

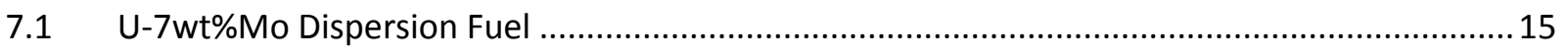

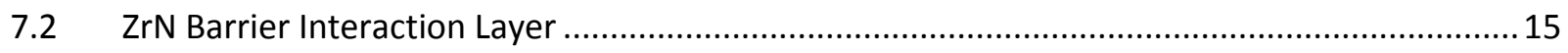

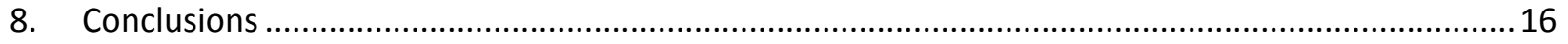

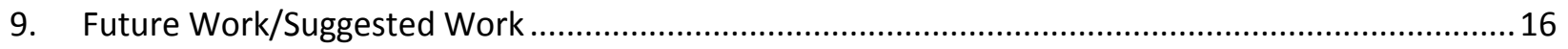

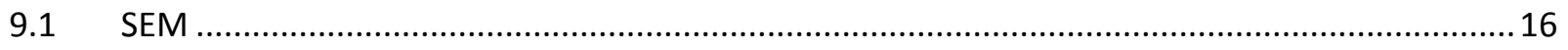

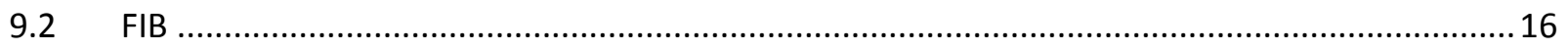

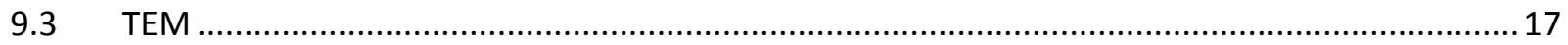

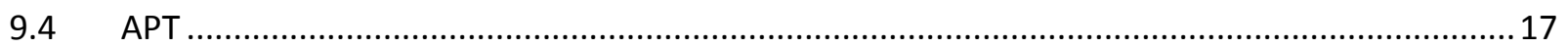

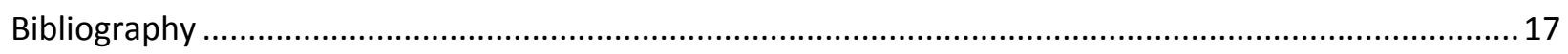

\section{List of Figures}

Figure 1: SEM micrographs of various cross-sections from plate U7MD1231 (2) ................................ 7

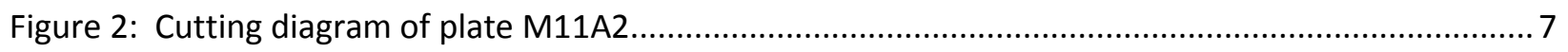

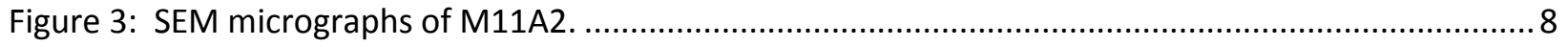

Figure 4: SEM micrograph showing an example of a cube on the copper grid. ...................................... 9

Figure 5: SEM micrograph showing the starting condition of the sectioning process. ............................ 9 
Figure 6: SEM micrographs showing various FIB slices of the $1^{\text {st }}$ cube of M11A2 ...............................10

Figure 7: SEM micrographs showing various FIB slices of the $2^{\text {nd }}$ cube of M11A2 .............................. 11

Figure 8: Two micrographs showing the curtaining artifact (left) and the processed image (left) devoid

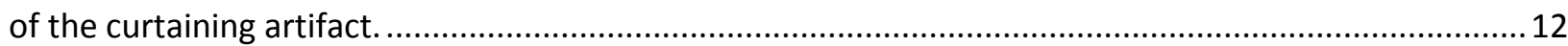

Figure 9: 3D reconstructions of the FIB/SEM images from the first cube showing the interaction layer (red), the ZrN layer (gold), Al-ZrN layer (blue), and Al matrix (green)................................................... 14

Figure 10: $3 \mathrm{D}$ recontructions of the FIB/SEM images from the $2^{\text {nd }}$ cube showing the interaction layer (red), ZrN (gold), Al-ZrN layer (grey), and U-Mo (blue) ....................................................................... 15

\section{List of Tables}

Table 1: SELENIUM plate average end of life irradiation parameters. 


\section{Acronyms:}

INL-Idaho National Laboratory

EML-Electron Microscopy Laboratory

EDS-Energy Dispersive Spectroscopy

FIB-Focused Ion Beam

EFPD-Effective Full Power Days

SEM-Scanning Electron Microscopy

ZrN-Zirconium Nitride 
INTENTIONALLY BLANK 


\section{Executive Summary}

Using one of the INL's FIBs, two cube of irradiated dispersion U-Mo fuel coated in ZrN were serial sectioned in 50 and $200 \mathrm{~nm}$ slices to create 3D reconstructions of the fuel microstructure. The purpose of the reconstructions were to show the "volcano" features formed when interaction layer grows at breaches in the protective ZrN layer. The 3D reconstruction was able to show morphology of multiple volcano features in cubes of irradiated fuel at a burnup of roughly $4 \times 10^{21} \mathrm{fiss} / \mathrm{cm}^{3}$. Most of the U-Mo fuel has not undergone recrystallization and shows fission gas pores decorating the grain boundaries. In areas where no breaches of the $\mathrm{ZrN}$ has occurred, the $\mathrm{ZrN}$ is useful in preventing Al interaction with the $\mathrm{U}-\mathrm{Mo}$ and forming traditional interaction layers seen in U-Mo dispersion fuels. The $\mathrm{Zr}$ rich $\mathrm{Al}$ matrix adjacent to the $\mathrm{ZrN}$ layer on the Al matrix side was seen. This is like the original $\mathrm{ZrN}$ layer that has decomposed with increasing burnup and has Al mixing occur.

\section{SELENIUM Background}

The SELENIUM experiment was designed to test the effects of Si and ZrN coatings on the irradiation behavior of U-Mo dispersion fuels. In particular, the prevention of interaction layer growth between the Al matrix and the U-Mo fuel particles. Two dispersion plates were irradiated in the experiment. One plate included Si coated fuel particles and additional information on this plate can be found in (1) (2). The other plate used ZrN coated fuel particles. The applied ZrN coating was approximately $1 \mu \mathrm{m}$ in thickness. The SELENIUM experiment was irradiated in the BR2 reactor at SCK.CEN for three cycles for a total of 68 effective full power days (EFPD). Microstructure characterization of the as received SELENIUM fuel plates can be found in (1). Table 1 provides the irradiation parameters of the SELENIUM experiment.

Table 1: SELENIUM plate average end of life irradiation parameters.

\begin{tabular}{|l|c|}
\hline Average ${ }^{235} \mathrm{U} \%$ Burnup & 48 \\
\hline Maximum ${ }^{235} \mathrm{U} \%$ Burnup & 70 \\
\hline Beginning of Life Power Density $\left(\mathrm{W} / \mathrm{cm}^{2}\right)$ & 470 \\
\hline
\end{tabular}

\section{Sample Preparation}

\subsection{SCK·CEN Sample Preparation}

Using neutronics from the plate, various sections of the ZrN fuel plate were sectioned to study the irradiated microstructure behavior at various fission densities. Section M11 was obtained from a fission density around 4.0 to $4.5 \times 10^{21} \mathrm{fiss} / \mathrm{cm}^{3}$. Figure 1 shows SEM micrographs that show the cross-section of the fuel at various fission densities (2). Figure 2 shows a diagram of where section M11 was sectioned into smaller sections. From Figure 2, section M11A2 was created and prepared for SEM and FIB analysis. The section was polished to a thickness of $\sim 150 \mu \mathrm{m}$ with a mirror finish on one side. The section is $\sim 2$ $\mathrm{mm}$ in length. Basic SEM was performed on the section and some results will be seen in the SEM characterization section. The sample was decontaminated and shipped to the INL for FIB analysis. 


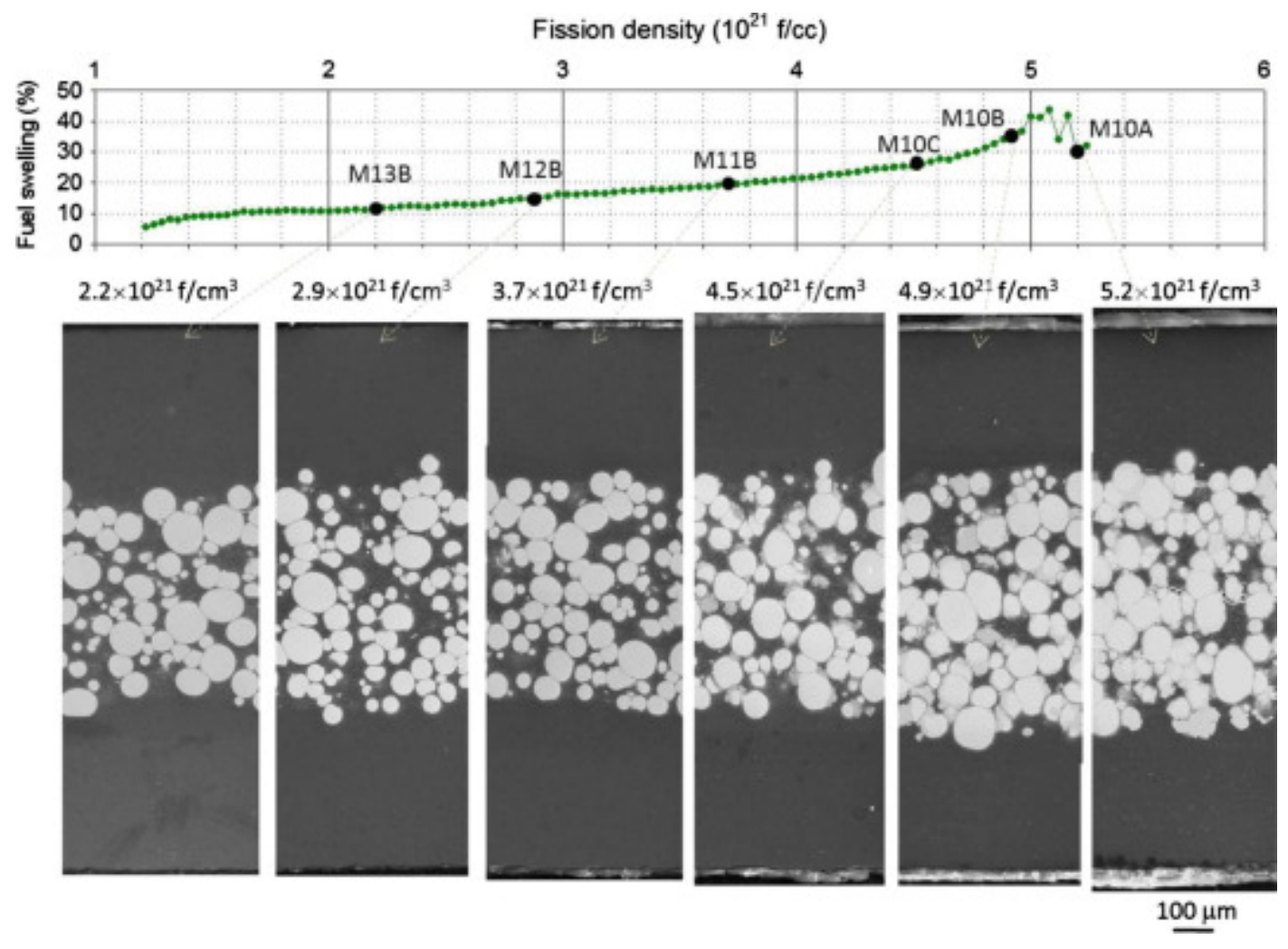

Figure 1: SEM micrographs of various cross-sections from plate U7MD1231 (2).

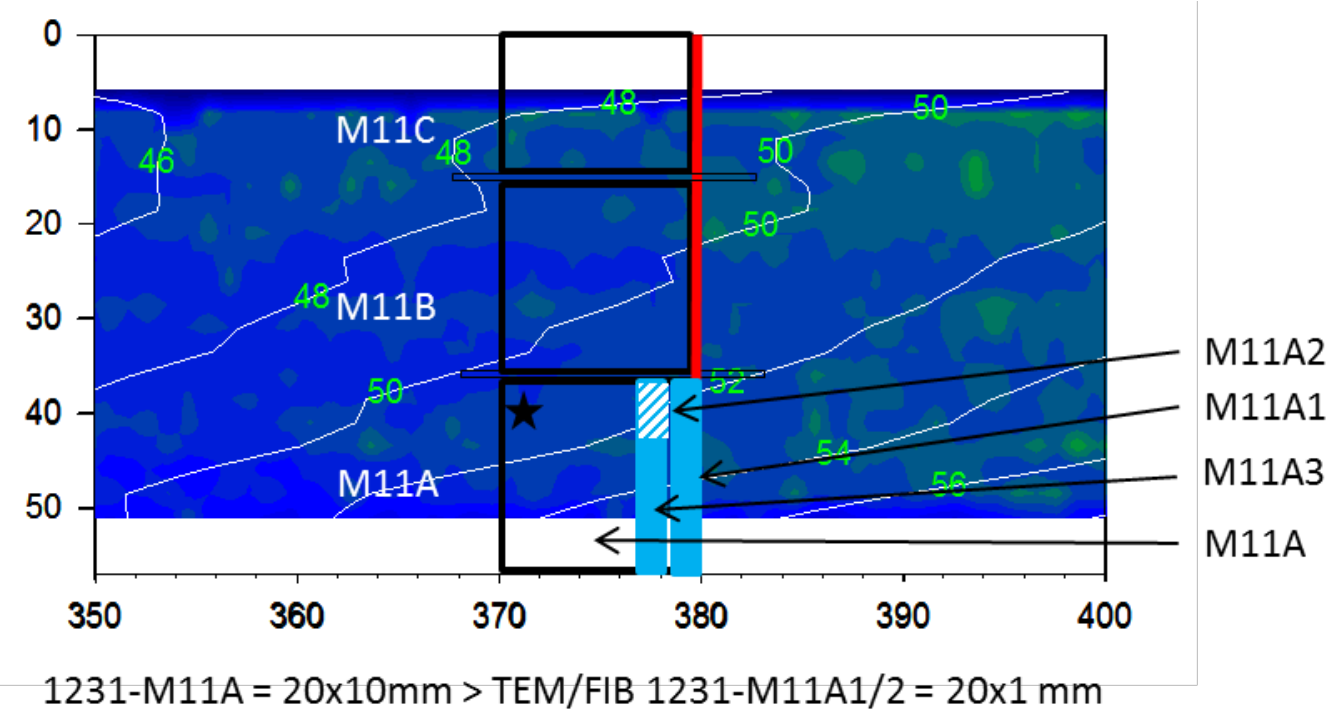

Figure 2: Cutting diagram of plate M11A2 


\subsection{Local Irradiation Conditions}

Local irradiation conditions for M11A2 were determined using the cutting diagram seen in Figure 2. Locally, M11A2 saw a $52 \%{ }^{235} \mathrm{U}$ burnup.

\subsection{EML Sample Preparation}

Sample M11A2 was loaded into an EML fume hood for mounting purposes. M11A2 was unloaded from its shipping container and was mounted onto a SEM stub with a sticky dot, polished side up.

Contamination levels were verified on M11A2. Once contamination levels reached benchtop allowable levels, a gold coat was applied to the sample surface and the sample was loaded into the FIB for characterization.

\section{SEM Characterization}

Basics SEM characterization of M11A2 was provided to the INL from SCK.CEN personnel to assist in the preparation of TEM and FIB samples. Figure 3 shows two SEM micrographs of sample M11A2. In Figure $3 b)$, the target volcano feature can be seen in the bottom left fuel kernel. Additional SEM characterization of the SELENIUM fuel plates can be seen in (2).
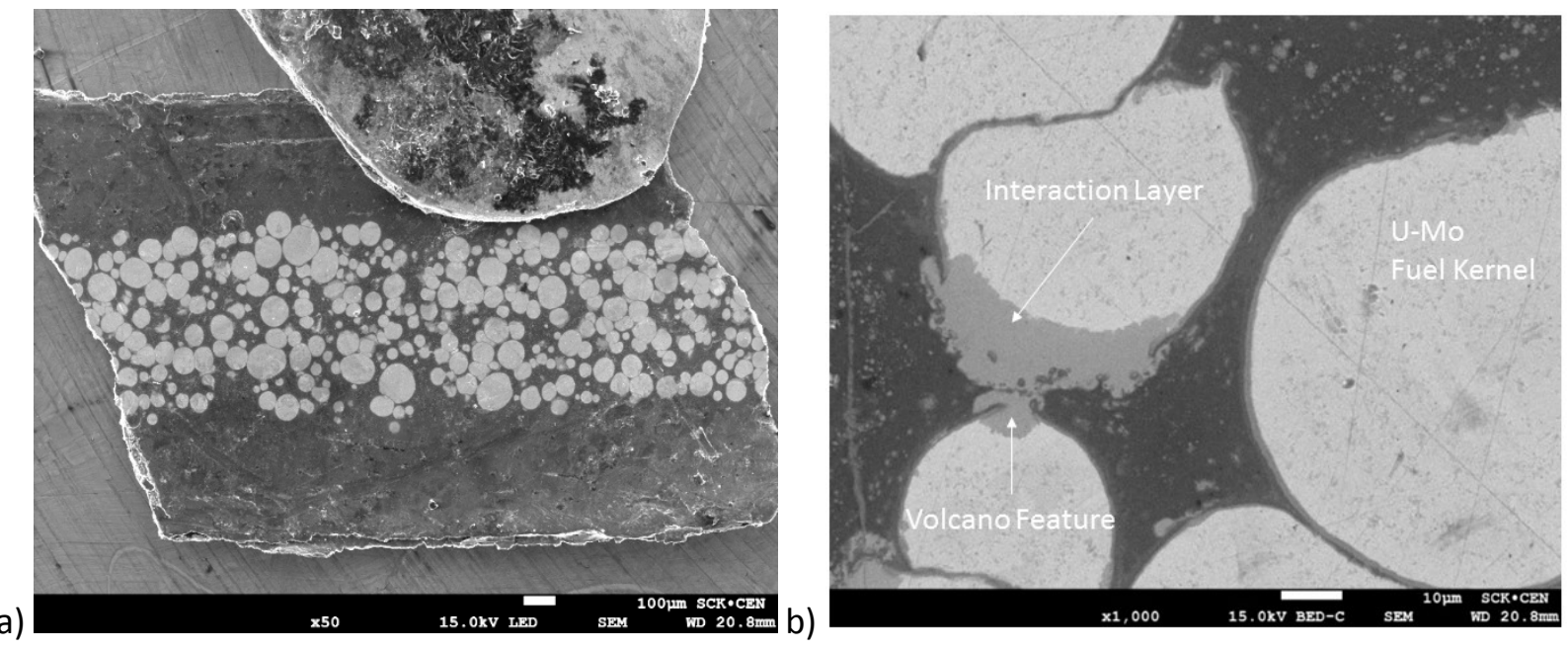

Figure 3: SEM micrographs of M11A2.

\section{FIB Characterization}

FIB was performed using an FEI Quanta 3D dual beam FIB in EML. TEM samples were created from M11A2 and were shipped to SCK.CEN for TEM analysis. For the 3D serial sectioning process, cubes roughly the size of $20 \times 20 \times 20 \mu \mathrm{m}$ of useable area were created using the FIB. Note that the due to the cutting process, there is a wedge shape on the bottom of the cubes that is used to release the cube from the host material and is the area where the cube is welded to the copper grid as not to disturb the useable area of the cube. Figure 4 shows an SEM micrograph that shows a cube attached to the copper grid for sectioning. 


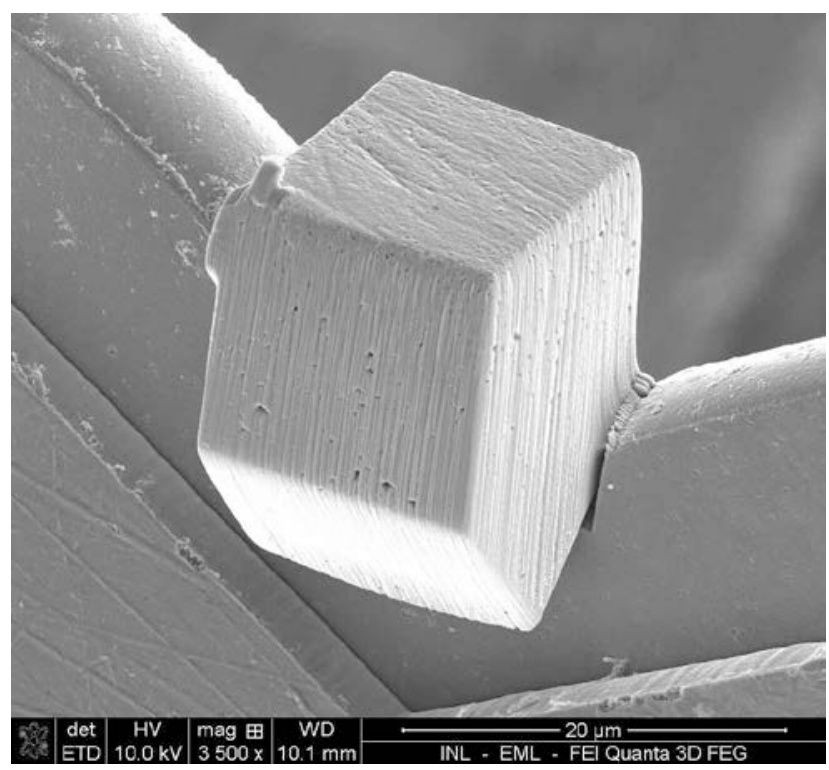

Figure 4: SEM micrograph showing an example of a cube on the copper grid.

In attempt to find the "volcano" feature in the FIB, two separate cubes were created from the host sample. The first cube was sectioned in roughly $200 \mathrm{~nm}$ slices. This large sectioning distance was due to time constraints in the FIB. Figure 5 shows the starting slice for the 3D reconstruction. The starting useable area is $22 \times 17.5 \mu \mathrm{m}$. Figure 6 shows various SEM micrographs of the FIB slices at different locations showing the evolution of "volcano" features in one of the M11A2 cubes.

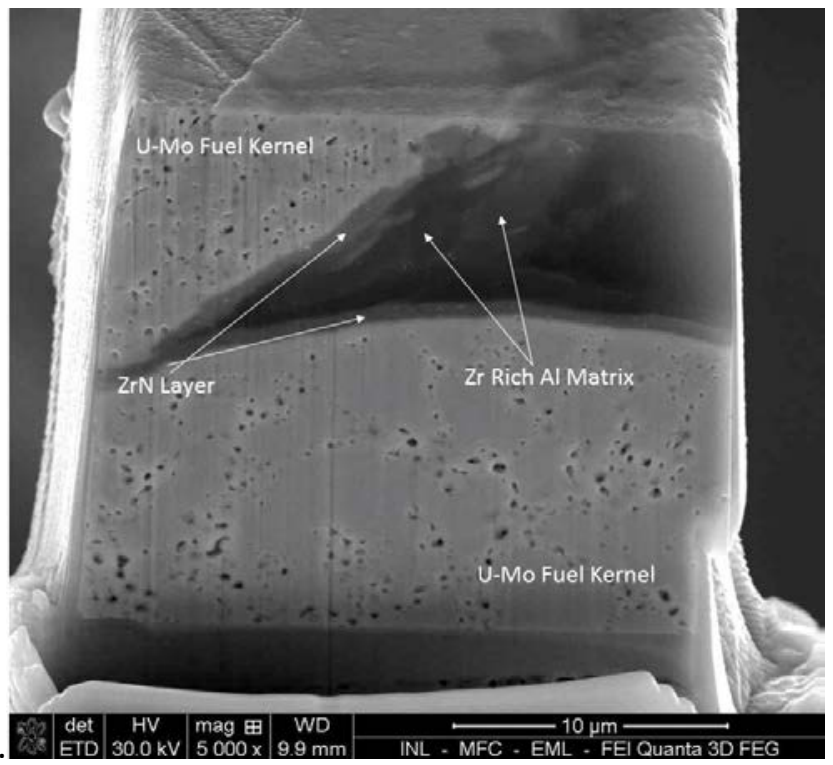

Figure 5: SEM micrograph showing the starting condition of the sectioning process. 
a)
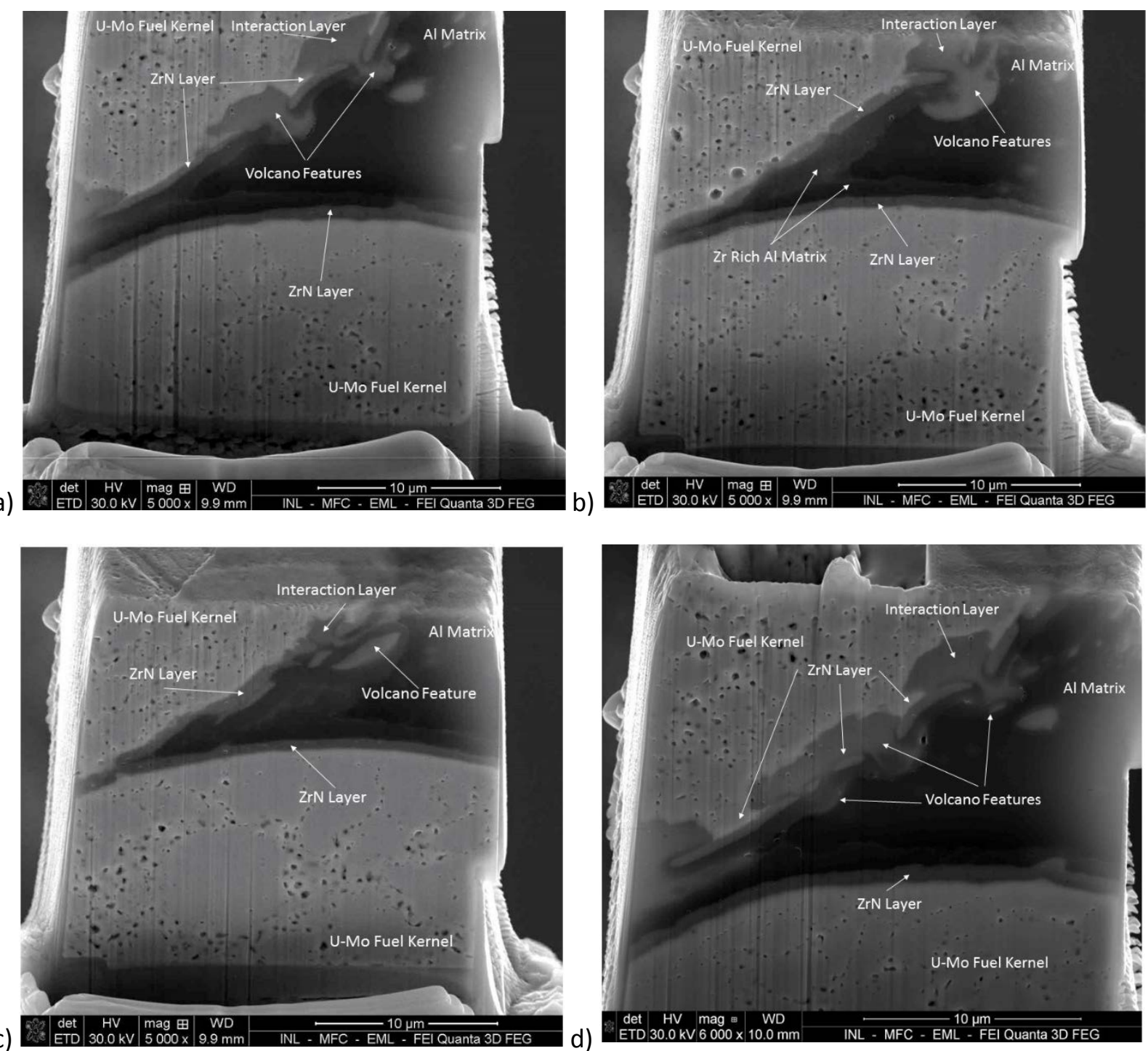

Figure 6: SEM micrographs showing various FIB slices of the $1^{\text {st }}$ cube of M11A2.

The general microstructure of the fuel region is as expected with fission gas bubbles decorating the residual fuel grains. As seen in the bottom fuel kernel in Figure $6 \mathrm{c}$ ), the $\mathrm{ZrN}$ layer around the bottom fuel kernel is preventing the Al matrix from interacting with the U-Mo fuel. When the ZrN layer has failed, as in the volcano feature areas, traditional interaction layer is formed. This layer can be present on the fuel side of the $\mathrm{ZrN}$ layer. Additionally, $\mathrm{U}$ and Mo appear to diffuse out of the U-Mo fuel kernel at the $\mathrm{ZrN}$ breach and form the "volcano" features seen in Figure 6. There is a light halo region towards the Al matrix on the outside of the ZrN layer. This is shown as the $\mathrm{Zr}$ rich Al matrix in Figure $6 \mathrm{~b})$. This area is $Z r$ rich nano crystalline Al. This has been confirmed in TEM results seen in (3). This layer was likely the original ZrN layer that has decomposed with increasing burnup likely due to the $A l$ that has mixed with the ZrN layer in this region and formed the Zr rich Al matrix layer.

For the second cube, $50 \mathrm{~nm}$ sections were used. This was done to provide better resultion between the slices to see features that may be smaller then the $200 \mathrm{~nm}$ slice thickness used in the $1^{\text {st }}$ cube. Figure 7 
shows various SEM micrographs of the sectioning of second cube of M11A2. As seen in the images, only one volcano feature was seen in this sectioning. This data set does still provide useful data on the behavior of the ZrN layer.
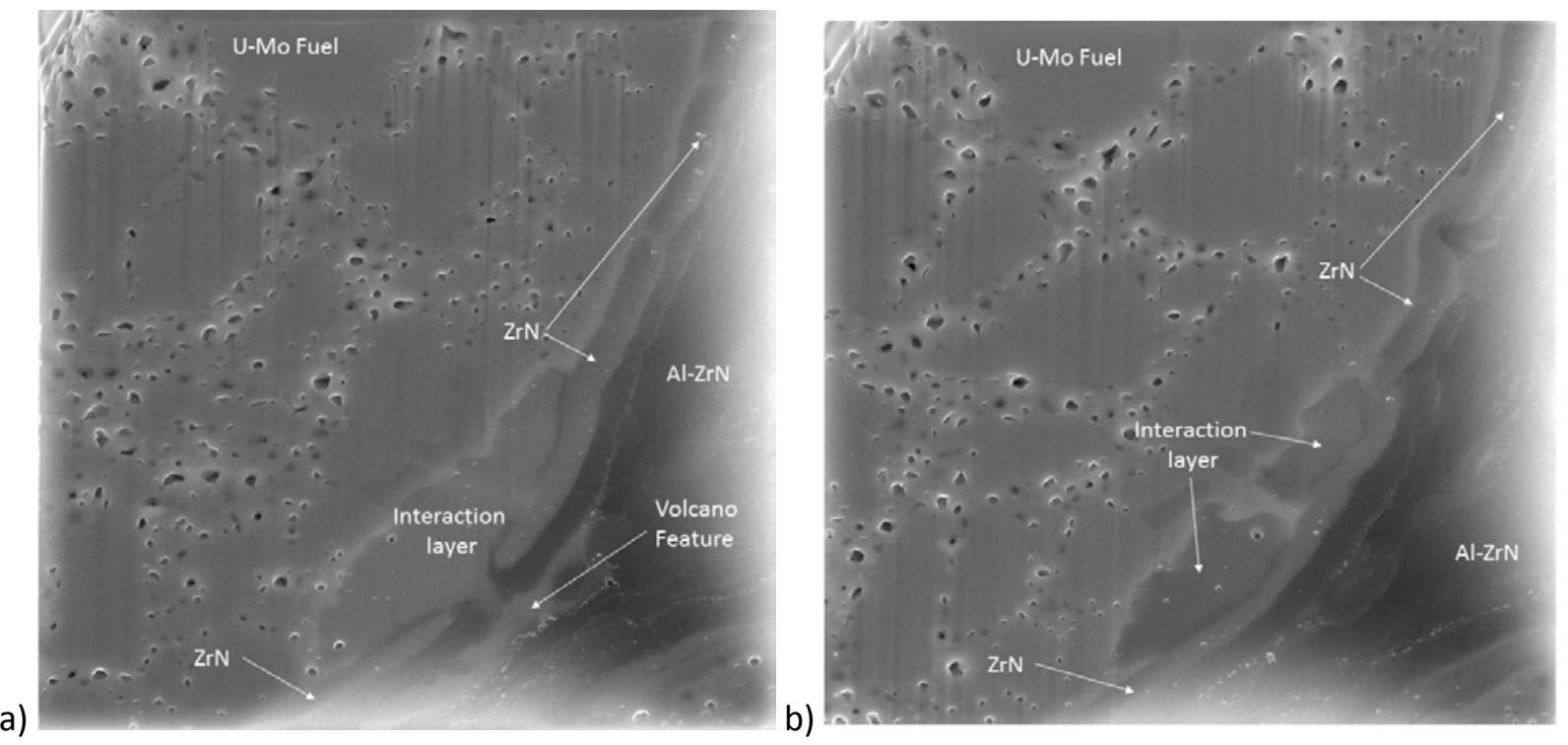

Figure 7: SEM micrographs showing various FIB slices of the $2^{\text {nd }}$ cube of M11A2.

\section{3D Reconstruction}

\subsection{Data Processing}

\subsubsection{Alignment correction}

Accurate image registration or image alignment is an essential step for 3D image reconstruction, by which images are spatially aligned (translated and/or rotated) to a reference. Two types of image registration, intensity-based and feature-based, can be used for images registration.

A 2D phase correlation determines a relative translative offset between two images. It is based on Fourier shifting property, which states that a shift in the coordinate frames of two functions is transformed in the Fourier domain as linear phase difference [5]. Fourier transform represents images in the frequency domain, which represents a phase difference between two images if they are shifted in the spatial domain.

If two images, $g(x, y)$ and $h(x, y)$, have simple translational shift ' $a$ ' in horizontal direction and ' $b$ ' in vertical direction, the Fourier transform of the two images is:

$$
H(u, v)=G(u, v) \mathrm{e}^{-i(a u+b v)}
$$

The normalized cross power spectrum is: WHAT IS L(u,v) 


$$
\mathrm{L}(u, v)=\frac{G(u, v) H(u, v)^{*}}{\left|G(u, v) H(u, v)^{*}\right|}=\mathrm{e}^{-i(a u+b v)}
$$

Taking the inverse of $L(u, v)$ leads to a Dirac delta function and its peak gives the shift between the two images.

\subsubsection{Frequency domain filtering}

The major problem that makes DualBeam FIB/SEM images difficult to analysis is the milling artifact, which also known as curtaining or waterfall effect. The local variations, e.g. voids or cracks, on the surface structure, affect the sputtering rates of the ion beam, which eventually creates these artifacts. Sample preparation, milling parameters, such as low beam current may reduce the curtaining effects, however, these may significantly increase in processing time for image acquisition. Moreover, for a specific sample geometry and microstructure, the curtaining effect may be unavoidable. Therefore, image filtering is a very important step for 3D reconstruction. These effects can be better seen in Figure 8. The left micrographshows volume of the fuel obtained by FIB/SEM images ( 55 slices, $200 \mathrm{~nm}$ thickness). The arrow shows the curtaining artifact on the image. The processed image does not contain cutaining artifacts.
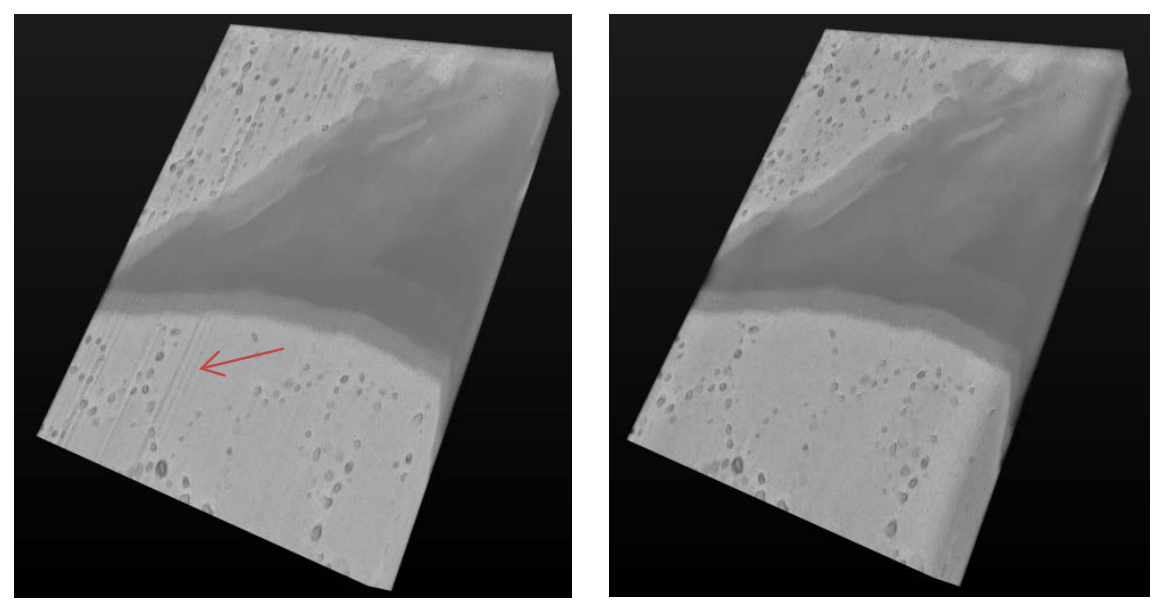

Figure 8: Two micrographs showing the curtaining artifact (left) and the processed image (left) devoid of the curtaining artifact.

The fast Fourier transform (FFT) filtering is an important image processing step for many applications. It computes the discrete Fourier transform (DFT) and decomposes the spatial data of an image into frequencies. Every pixel in the Fourier domain corresponds to a spatial frequency value, in which the magnitude of the value corresponds to the brightness of the pixel. The abrupt intensity variation corresponds to the high frequencies and the gradual transition corresponds to the low frequencies in 
the Fourier image. The DC- term that appears at the center of the Fourier image provides the average brightness. The convolution in spatial domain corresponds to the simple multiplication in the Fourier domain. Once the FFT filter is constructed, the image can be processed in many ways. Using different filters, certain frequencies can be retained or discarded. The curtaining artifacts are typically oriented in parallel to the milling direction and appear horizontally in the Fourier image. Removing the frequencies along the horizontal direction and then inverting the FFT removes the curtaining artifacts from the FIB/SEM images.

\subsection{D Recontruction Results}

Intel Xeon CPU E5-2630 2.40 GHz with 192 GB RAM, NVIDIA Quadro K6000 graphics card and 64- bit operating system of Windows 7 was used to perform image processing and 3D reconstructions. Material Image Processing and Automated Reconstruction (MIPAR) was used to perform automated image registration and pre-processing. After processing the dateset using MIPAR $^{\mathrm{TM}}$, the Avizo ${ }^{\mathrm{TM}}$ software is used to perform 3D reconstruction and segmentation of the features.

Figure 9 shows results of 3D reconstruction of the $1^{\text {st }}$ cube ( $200 \mathrm{~nm}$ slices). Cracks in the ZrN layer can be easily noticed in the images, providing the pathway for diffusion of $\mathrm{U}, \mathrm{Mo}$, and $\mathrm{Al}$ and creating the traditional interaction layer that forms in U-Mo/Al matrix fuels. What is interesting is the ZrN layer that shows no cracking or interaction layer formation. The layer shows relativiely consistant thickness thoughout with a few peaks throughout where extra deposition occurred. This indicates that the deposition process is fairly consistant and the ZrN's irradiation performance appears to be stable, though cracks do appear to fail in various locations, causing the "volcano feature".
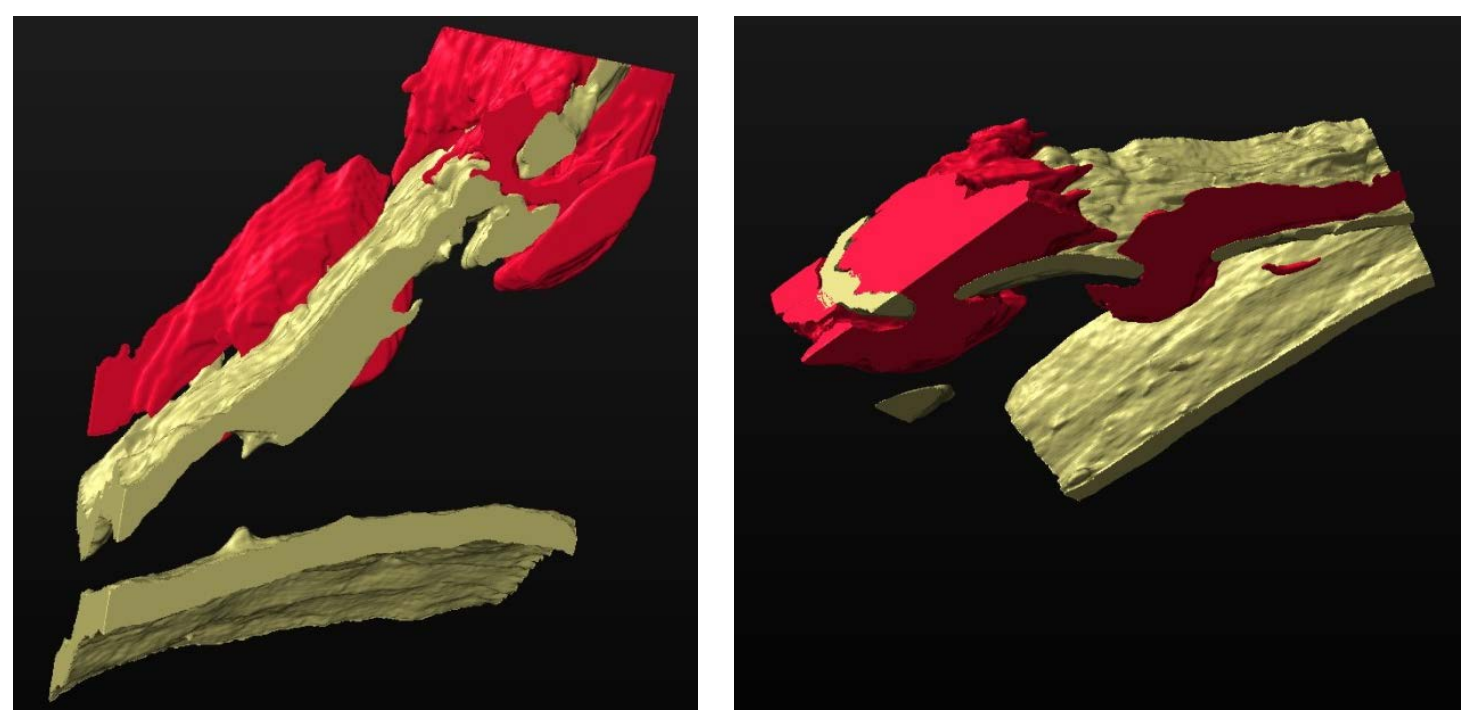

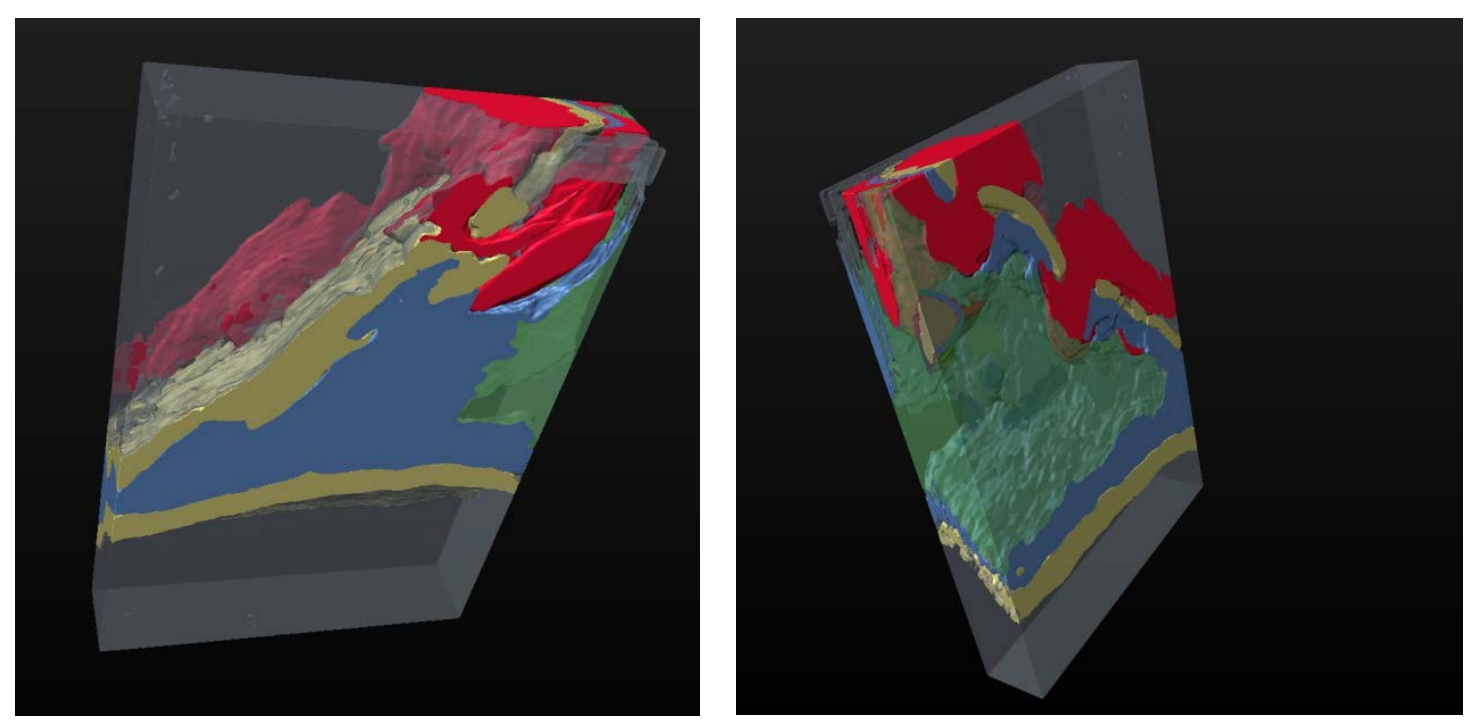

Figure 9: 3D reconstructions of the FIB/SEM images from the first cube showing the interaction layer (red), the ZrN layer (gold), Al-ZrN layer (blue), and Al matrix (green).

Figure 10 shows 3D reconstructions of the $2^{\text {nd }}$ cube. Though less volcano features are seen in this reconstruction, the one present in this reconstruction shows clearly the formation of the volcano feature on the Al side of the crack in the $\mathrm{ZrN}$. On the fuel side of the crack, the interaction layer diffuses away from the crack in all directions, even if there is intact ZrN present between the fuel and Al matrix. With this diffusion of the interaction layer away from the crack in the fuel side, interpretation of SEM data that showed interaction layer growth but no cracks is solved. It is likely that a crack is present below or above the plane that the SEM characterization was being performed. 

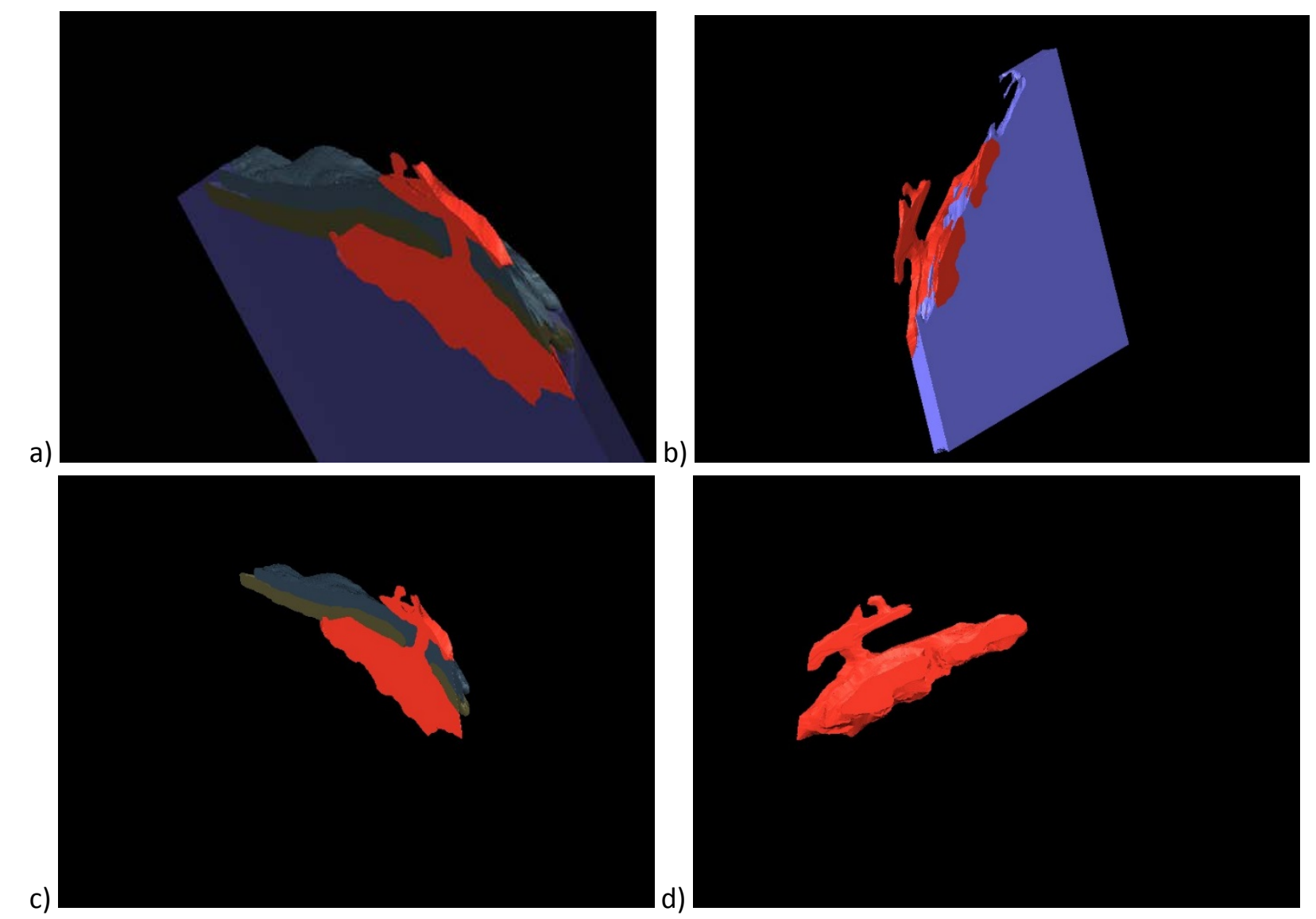

Figure 10: 3D recontructions of the FIB/SEM images from the $2^{\text {nd }}$ cube showing the interaction layer (red), ZrN (gold), Al-ZrN layer (grey), and U-Mo (blue)

\section{Discussion}

\subsection{U-7wt\%Mo Dispersion Fuel}

The 3D reconstruction of the SEM micrographs show that the irradiated U-7Mo fuel behaves as expected. Fuel grains are present throughout with fission gas bubbles decorating the grain boundaries, common traits of an irradiation burnup of less than $4.5 \times 10^{21} \mathrm{fiss} / \mathrm{cm}^{3}$. Using the SEM sectioning results, the intergranular bubble sizes distributions will be calculated at various sections at a future date. TEM characterization results will be published at a future date.

\subsection{ZrN Barrier Interaction Layer}

The $\mathrm{ZrN}$ barrier appears to prevent interaction layer growth in most areas of the fuel kernels. In areas where a crack in the ZrN layer has occurred, interaction layer has formed. The interaction layer forms into the U-Mo fuel kernel and out towards the Al matrix, indicating that $U$ and Mo diffuse/mix out of the $\mathrm{U}-\mathrm{Mo}$ fuel kernel and $\mathrm{Al}$ into the U-Mo fuel kernel. The interaction layer does form behind the $\mathrm{ZrN}$ layer, diffusing from from the crack in the ZrN layer. It is not clear how prevalent the failure of the $\mathrm{ZrN}$ layer is in the fuel. It would interesting to take a large cross-section of the fuel plate, polish it to a few 
different planes, examine the volcano features at each plan, and get a better idea of how many failures are occurring.

Another variable in the failure of the $\mathrm{ZrN}$ coating is the burnup that the fuel has undergone. As seen in Figure $6 \mathrm{~b}$ ), there is a $\mathrm{Zr}$ rich Al matrix region that has formed on the outside of the ZrN layer towards the Al matrix. This layer seems to be effected by the fission density in the material. Characterization of the fresh fuel does not show this layer forming with only the $\mathrm{ZrN}$ layer present in a thickness of $\sim 1 \mu \mathrm{m}$ (4). The thickness of the Al-Zr rich region and the ZrN layer is approximetly the same size as the original ZrN layer thickness.

This decrease in ZrN layer thickness could lead to cracking of the ZrN layer due to stresses in the fuel and matrix. With increasing burnup, the swelling in the U-Mo will increase due to solid and gaseous fission products. This increased volume will lead to an outward radially stress on the ZrN layer, most likely causing breaches/cracks. These cracks then offer an increased diffusion pathway for the Al, Mo, and $U$ interaction layer.

This method of using 3D reconstructions appears to be useful tool in interpretating physical mechinisms occurring in a material. It was not clear if the Al was diffusing through the $\mathrm{ZrN}$ or through cracks in the $\mathrm{ZrN}$. This work shows that the interaction layer indeed comes from defects in the ZrN layer such as cracks. An intact ZrN layer has been shown to prevent interaction layer growth between the U-Mo fuel and the Al matrix.

\section{Conclusions}

Two serial sections were performed on U-Mo dispersion fuel coated with ZrN. The U-Mo fuel had undergone a $52 \% \mathrm{U}^{235}$ burnup. SEM imaging at SCK.CEN showed the formation of intereaction in breaches in the $\mathrm{ZrN}$ and in various regions of the U-Mo fuel in regions where no ZrN breach was evident. Serial sectioning was performed to understand if the interaction layer seen in the U-Mo behind the ZrN was from a breach above or below the surface. Serial sectioning showed that interaction layer diffused through the breachs in the ZrN layer. Multiple breaches were seen in two serial sections performed. In regions that the $\mathrm{ZrN}$ is intact, the layer is fairly consistant in size. The serial sectioning process appears to be of use with nuclear fuels. The ability to characterize features in a 3D manner provides useful information about how the features form, cluster, and/or behave.

\section{Future Work/Suggested Work}

\subsection{SEM}

None seen at the current time.

\section{$9.2 \quad$ FIB}

Currently, there is one more cubes that are available for slicing. This cube is still attached to the host material and requires minimal time to release the cube and attach it to a copper grid. A suggested idea 
for the remaining cube is to obtain EDS profiles at each slice as well to provide both images and chemical segregation in each slice.

\subsection{TEM}

None seen at the current time.

\subsection{APT}

None seen at the current time.

\section{Bibliography}

1. Surface engineering of low enriched uranium-molybdenum. A. Leenaers, S. Van den Berge, C. Detavernier. 2013, Journal of Nuclear Materials, Vol. 440, pp. 220-228.

2. Fuel Swelling and interaction layer formation in the SELENIUM Si and ZrN coated U(Mo) dispersion fuel plates irradiated at high bower in BR2. A. Leenaers, S. Van den Berghe, E. Koonen, V. Kuzminov, C. Detavernier. 2015, Journal of Nuclear Materials, Vol. 458, pp. 380-393.

3. Post irradiation TEM investigation of ZrN coated $U(M o)$ particles prepared with FIB. W. Van Renterghem, A. Leenaers, S. Van den Berghe, B. D. Miller, J. Gan, J. W. Madden, D. D. Keiser Jr. Seoul, South Korea : s.n., Oct. 11-14, 2015, RERTR 2015 36th INternational Meetin on Reduced Rerichment for Research and Test Reactors.

4. Surface engineering of low enriched uranium-molybdenum. A. Leenaers, S. Van den Berghe, C. Detavernier. 20013, Journal of Nuclear Materials, Vol. 440, pp. 220-228.

5. Kamel, Mohamed and Campilho, Aurelio. Image Analysis and Recognition. s.I. : Springer, 2013. 